\title{
Wave Interactions of the Aw-Rascle Model for Generalized Chaplygin Gas*
}

\section{Yujin Liu}

School of Mathematics and Statistics, Shandong University of Technology, Zibo, China

Email: yjliu98@126.com

How to cite this paper: Liu, Y.J. (2021) Wave Interactions of the Aw-Rascle Model for Generalized Chaplygin Gas. Journal of Applied Mathematics and Physics, 9, 317-327.

https://doi.org/10.4236/jamp.2021.92023

Received: January 11, 2021

Accepted: February 23, 2021

Published: February 26, 2021

Copyright (c) 2021 by author(s) and Scientific Research Publishing Inc. This work is licensed under the Creative Commons Attribution International License (CC BY 4.0).

http://creativecommons.org/licenses/by/4.0/

\begin{abstract}
In this paper, we investigate the elementary wave interactions of the Aw-Rascle model for the generalized Chaplygin gas. We construct the unique solution by the characteristic analysis method and obtain the stability of the corresponding Riemann solutions under such small perturbations on the initial values. We find that the elementary wave interactions have a much more simple structure for Temple class than general systems of conservation laws. It is important to study the elementary waves interactions of the traffic flow system for the generalized Chaplygin gas not only because of their significance in practical applications in the traffic flow system, but also because of their basic role for the general mathematical theory.
\end{abstract}

\section{Keywords}

Wave Interaction, Aw-Rascle Model, Generalized Chaplygin Gas, Riemann Problem, Delta Shock, Hyperbolic Conservation Laws

\section{Introduction}

In the present paper, we study the Aw-Rascle (AR) macroscopic model of traffic flow

$$
\left\{\begin{array}{l}
\rho_{t}+(\rho u)_{x}=0, \\
(\rho(u+P))_{t}+(\rho u(u+P))_{x}=0,
\end{array}\right.
$$

where $\rho \geq 0$ is the density, $u \geq 0$ is the velocity, $P$ is the velocity offset which is called as the "pressure" inspired from gas dynamics. The derivation process of the above AR model and the application can be discovered in [1]-[7].

In [8], Aw and Rascle studied the limit behavior and found that the pressure term is active. In [9], Shen and Sun investigated the limit behavior without the 
constraint of the maximal density.

In [10] [11], M. N. Sun studied the following model

$$
\left\{\begin{array}{l}
\rho_{t}+(\rho u)_{x}=0, \\
\left(\rho u+\rho^{\gamma}\right)_{t}+\left(\rho u^{2}+\rho^{\gamma} u\right)_{x}=0,
\end{array}\right.
$$

In [10] [11], they studied the elementary wave interactions and obtained the stability of the Riemann solutions under such a perturbation on the initial data.

In [12], G. D. Wang investigated the Riemann problem of (1) and

$$
p=-\frac{B}{\rho^{\alpha}},
$$

where $0<\alpha \leq 1, B>0$. This is the so-called generalized Chaplygin gas for (1).

In the present paper, we investigate the elementary wave interactions for (1) and (3). In our paper [13], we study the wave interactions containing no delta shock, so we just consider the wave interactions for (1) containing delta shock wave with the three piecewise constants

$$
(u, \rho)(x, 0)= \begin{cases}\left(u_{-}, \rho_{-}\right), & -\infty<x<-\varepsilon, \\ \left(u_{m}, \rho_{m}\right), & -\varepsilon<x<\varepsilon, \\ \left(u_{+}, \rho_{+}\right), & \varepsilon<x<+\infty,\end{cases}
$$

where the perturbation parameter $\varepsilon$ is sufficiently small. (4) can be regarded as a local perturbation on the initial values

$$
(u, \rho)(x, 0)=\left(u_{ \pm}, \rho_{ \pm}\right), \quad \pm x>0,
$$

where $u_{ \pm}, \rho_{ \pm}>0$.

This paper is arranged as follows. In Section 2, we give curtly the Riemann problem for the model (1) (3) and (5) for the convenience of the readers. In Section 3 , we investigate the elementary wave interactions by the characteristic analysis method. In Section 4, we summarize our main conclusion.

\section{Preliminaries}

We give briefly the Riemann problem for (1) (3) and (5) [12].

The characteristic roots of (1) are $\mu_{1}=u-\frac{B \alpha}{\rho^{\alpha}}, \mu_{2}=u$ which shows that (1) is strictly hyperbolic. The corresponding right characteristic vector of $\mu_{1}$ and $\mu_{2}$ is respectively given by

$$
\boldsymbol{v}_{1}=\left(-\frac{B \alpha}{\rho^{1+\alpha}}, 1\right)^{\mathrm{T}}, \quad \boldsymbol{v}_{2}=(0,1)^{\mathrm{T}},
$$

If $0<\gamma<1$, we get

$$
\nabla \mu_{1} \cdot \boldsymbol{v}_{1}=-\frac{(1-\alpha) B \alpha}{\rho^{1+\alpha}}<0, \quad \nabla \mu_{2} \cdot \boldsymbol{v}_{2}=0,
$$


which indicates that $\mu_{1}$ is genuinely nonlinear and the associated wave is either shock wave or rarefaction wave, $\mu_{2}$ is always linearly degenerate and the associated wave is the contact discontinuity, where $\nabla$ denotes the gradient.

We construct the self-similar solution $(u, \rho)(x, t)=(u, \rho)(\zeta), \zeta=\frac{x}{t}$. The Riemann problem (1) (5) becomes the following boundary value problem of the ordinary differential equations

$$
\left\{\begin{array}{l}
-\zeta \rho_{\zeta}+(\rho u)_{\zeta}=0 \\
-\zeta\left(\rho u-\frac{B}{\rho^{\alpha-1}}\right)_{\zeta}+\left(\rho u^{2}-\frac{u B}{\rho^{\alpha-1}}\right)_{\zeta}=0
\end{array}\right.
$$

and $(u, \rho)( \pm \infty)=\left(u_{ \pm}, \rho_{ \pm}\right)$. For smooth solutions, let $V=(u, \rho)^{\mathrm{T}},(8)$ becomes

$$
A(V) V_{\zeta}=0
$$

where

$$
A(V)=\left(\begin{array}{cc}
\rho & u-\zeta \\
u-\frac{B \alpha}{\rho^{\alpha}}-\zeta & 0
\end{array}\right) .
$$

Besides the constant state solution $(u, \rho)=$ constant, (9) has a rarefaction wave solution. For the given left state $\left(u_{-}, \rho_{-}\right)$, the rarefaction wave curve is given by

$$
R\left(u_{-}, \rho_{-}\right):\left\{\begin{array}{l}
\zeta=\lambda_{1}=u-\frac{\alpha B}{\rho^{\alpha}}, \\
u=u_{-}+\frac{B}{\rho^{\alpha}}-\frac{B}{\rho_{-}^{\alpha}}, \\
\rho<\rho_{-}, u>u_{-} .
\end{array}\right.
$$

For a bounded discontinuity at $\zeta=\tau$, it holds the Rankine-Hugoniot conditions

$$
\left\{\begin{array}{l}
-\tau[\rho]+[\rho u]=0 \\
-\tau\left[\rho u-\frac{B}{\rho^{\alpha-1}}\right]+\left[\rho u^{2}-\frac{u B}{\rho^{\alpha-1}}\right]=0,
\end{array}\right.
$$

where $[\rho]=\rho_{r}-\rho_{l}, \rho_{l}=\rho(\tau-0), \rho_{r}=\rho(\tau+0)$, etc.

For the given left state $\left(u_{-}, \rho_{-}\right)$, the shock wave is given by

$$
S\left(u_{-}, \rho_{-}\right):\left\{\begin{array}{l}
\tau=u-\frac{B}{\rho^{\alpha}}-\frac{\left(\rho_{-}^{1-\alpha}-\rho^{1-\alpha}\right) B}{\rho-\rho_{-}}, \\
u=u_{-}+\frac{B}{\rho^{\alpha}}-\frac{B}{\rho_{-}^{\alpha}} \\
\rho>\rho_{-}, u<u_{-} .
\end{array}\right.
$$

Since $\lambda_{2}$ is linearly degenerate, from (9) or (11) we know the contact discontinuity

$$
J: \zeta=u=u_{-}
$$


All the above rarefaction waves $R$, shock waves $S$, and contact discontinuities $J$ are the elementary waves for (1). Notice the shock curves coincide with the rarefaction curves in the phase plane $(u, \rho)$ [14]. It is very important because it can simplify the process of the elementary wave interactions.

According to the right state $\left(u_{+}, \rho_{+}\right)$in the different region (Figure 1 ), we obtain the unique Riemann solution. When $\left(u_{+}, \rho_{+}\right) \in \mathrm{I}$ or II, the unique Riemann solution is $R+J$, when $\left(u_{+}, \rho_{+}\right) \in \mathrm{III}$ or IV, the unique Riemann solution is $S+J$, when $\left(u_{+}, \rho_{+}\right) \in \mathrm{V}$, i.e., $u_{+}<u_{-}-\frac{B}{\rho_{-}^{\alpha}}<u_{-}$, we should construct the delta shock wave solution as follows.

Consider a piecewise smooth solution of (1) with the form

$$
(u, \rho)(x, t) \begin{cases}\left(u_{-}, \rho_{-}\right), & x<\tau t, \\ \left(u_{\delta}, \beta(t) \delta(x-\sigma t)\right), & x=\tau t \\ \left(u_{+}, \rho_{+}\right), & x>\tau t,\end{cases}
$$

where

$$
\begin{aligned}
& \tau=u_{\delta}=\frac{[2 \rho u+\rho p]+\sqrt{[\rho p]^{2}+4 \rho_{+} \rho_{-}[u][u+p]}}{2[\rho]}, \\
& \beta(t)=\left([\rho] u_{\delta}-[\rho u]\right) t .
\end{aligned}
$$

$\delta$-measure solutions $(u, \rho)$ of (1) (3) and (5) is given

$$
u(x, t)=u_{-}+[u] H(x-\sigma t), \quad \rho(x, t)=\rho_{-}+[\rho] H(x-\sigma t)+\beta(t) \delta_{L},
$$

here $L=\{(\sigma t, t): 0 \leq t<+\infty\}, H(x)$ is the Heaviside function.

The measure solution (14) and (15) satisfies the generalized Rankine-Hugoniot condition

$$
\left\{\begin{array}{l}
\frac{\mathrm{d} x}{\mathrm{~d} t}=u_{\delta}(t), \\
\frac{\mathrm{d} \beta(t)}{\mathrm{d} t}=[\rho] u_{\delta}(t)-[\rho u], \\
\frac{\mathrm{d}\left(\beta(t) u_{\delta}(t)\right)}{\mathrm{d} t}=[\rho(u+P)] u_{\delta}(t)-[\rho u(u+P)],
\end{array}\right.
$$

where $[u]=u(x(t)+0)-u(x(t)-0)$ is the jump of $u$ across the discontinuity $x=x(t)$, etc.

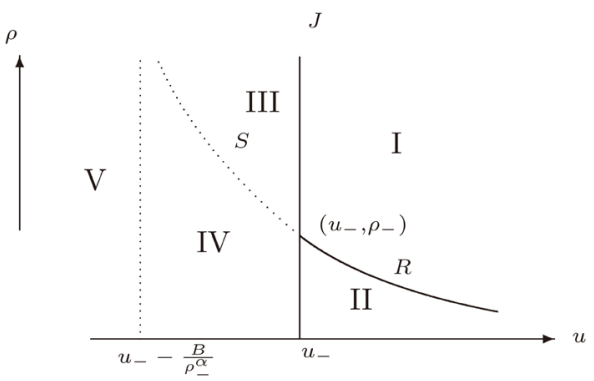

Figure 1. Wave curves in $(u, \rho)$. 
The $\delta$-entropy condition is

$$
\lambda_{1}\left(u_{+}, \rho_{+}\right) \leq \lambda_{2}\left(u_{+}, \rho_{+}\right) \leq u_{\delta} \leq \lambda_{1}\left(u_{-}, \rho_{-}\right) \leq \lambda_{1}\left(u_{-}, \rho_{-}\right),
$$

which is

$$
u_{+}-\frac{B \alpha}{\rho_{+}^{\alpha}} \leq u_{+} \leq u_{\delta} \leq u_{-}-\frac{B \alpha}{\rho_{-}^{\alpha}} \leq u_{-} .
$$

We know that all the characteristics on both sides of the $\delta$-shock wave curve are incoming.

In order to consider the interaction of elementary waves containing delta shock wave, we briefly recall the concept of left- and right-hand side delta functions as follows.

Let $\overline{R_{+}^{2}}$ be divided into two disjoint open sets $\Omega_{1}$ and $\Omega_{2}$ with a piecewise smooth boundary curve $L$, which satisfies $\Omega_{1} \cap \Omega_{2}=\varnothing$ and $\overline{\Omega_{1}} \cup \overline{\Omega_{2}}=\overline{R_{+}^{2}}$. Let $\mathcal{C}\left(\Omega_{i}\right)$ and $\mathcal{M}\left(\Omega_{i}\right)$ be the space of bounded and continuous real-valued functions equipped with the $L^{\infty}$-norm and the space of measures on $\Omega_{i}(i=1,2)$, respectively. Let us assume that $\mathcal{C}_{L}=\left(\mathcal{C}\left(\Omega_{1}\right), \mathcal{C}\left(\Omega_{2}\right)\right)$ and $\mathcal{M}_{L}=\left(\mathcal{M}\left(\Omega_{1}\right), \mathcal{M}\left(\Omega_{2}\right)\right)$, then the product of $G=\left(G_{1}, G_{2}\right) \in \mathcal{C}_{L}$ and $D=\left(D_{1}, D_{2}\right) \in \mathcal{M}_{L}$ is defined as an element $G D=\left(G_{1} D_{1}, G_{2} D_{2}\right) \in \mathcal{M}_{L}$, where $G_{i} D_{i}(i=1,2)$ can be defined as the usual product of a continuous function and a measure. Thus, it is obvious that the above-defined product makes sense.

We view the measure on $\overline{\Omega_{i}}$ as a measure on $\overline{R_{+}^{2}}$ with support in $\overline{\Omega_{i}}(i=1,2)$. Then the mapping $m: M_{L} \rightarrow M\left(\overline{R_{+}^{2}}\right)$ can be obtained by taking $m(D)=D_{1}+D_{2}$. In a similar way, we have $m(G D)=G_{1} D_{1}+G_{2} D_{2}$. The solution concept used in our paper when we consider the delta shock can be described as follows: carry out the multiplication and composition in the space $M_{L}$ and take the mapping $m: M_{L} \rightarrow M\left(\overline{R_{+}^{2}}\right)$ before differentiation in the space of distributions.

Based on the above analysis, we have the following conclusion.

Theorem 2.1 The Riemann solution of the initial value problem (1) (3) and (5) is unique.

\section{Interactions of Elementary Waves Containing Delta Shock Wave}

Now we study the elementary wave interactions for (1) (3) with (4). (4) is regarded as the perturbation on the Riemann initial values (5). In order to cover all the cases containing delta shock completely, we have three possibilities according to the different combinations from $(-\varepsilon, 0)$ and $(\varepsilon, 0)$ as follows: $\delta S$ and $\delta S, S+J$ and $\delta S, R+J$ and $\delta S$.

Case 1: $\delta S_{1}$ and $\delta S_{2}$.

We consider the interaction of a delta shock wave $\delta S_{1}$ emanating from $(-\varepsilon, 0)$ and a delta shock wave $\delta S_{2}$ emanating from $(\varepsilon, 0)$. When $t$ is small enough, the solution of the initial value problem (1) (3) and (4) is expressed as (Figure 2). 


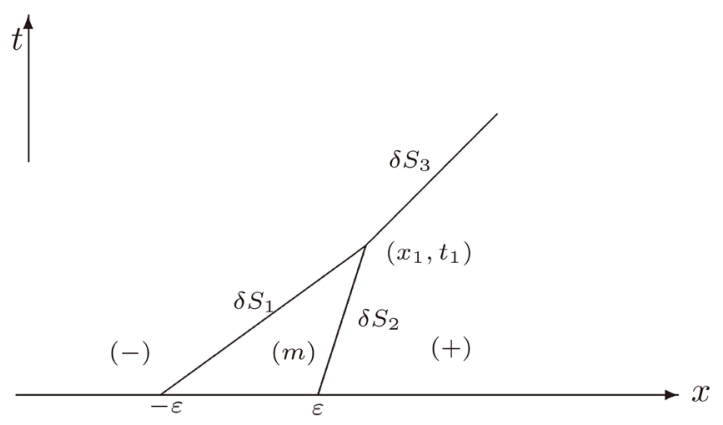

Figure 2. Interaction of $\delta S_{1}$ and $\delta S_{2}$.

$$
\left(u_{-}, \rho_{-}\right)+\delta S_{1}+\left(u_{m}, \rho_{m}\right)+\delta S_{2}+\left(u_{+}, \rho_{+}\right),
$$

The propagation speed of $\delta S_{1}$ and $\delta S_{2}$ satisfy respectively the $\delta$-entropy conditions

$$
\begin{aligned}
& u_{m}-\frac{B \alpha}{\rho_{m}^{\alpha}}<u_{m}<\tau_{\delta_{1}}<u_{-}-\frac{B \alpha}{\rho_{-}^{\alpha}}<u_{-}, \\
& u_{+}-\frac{B \alpha}{\rho_{+}^{\alpha}}<u_{+}<\tau_{\delta_{2}}<u_{m}-\frac{B \alpha}{\rho_{m}^{\alpha}}<u_{m},
\end{aligned}
$$

and we know that

$$
\begin{gathered}
\tau_{\delta_{1}}=\frac{\left(2 \rho_{m} u_{m}+\rho_{m} P_{m}-2 \rho_{-} u_{-}-\rho_{-} P_{-}\right)+\sqrt{\left(\rho_{m} P_{m}-\rho_{-} P_{-}\right)^{2}+4 \rho_{-} \rho_{m}\left(u_{m}-u_{-}\right)\left(u_{m}+P_{m}-u_{-} P_{-}\right)}}{2\left(\rho_{m}-\rho_{-}\right)}, \\
\tau_{\delta_{2}}=\frac{\left(2 \rho_{+} u_{+}+\rho_{+} P_{+}-2 \rho_{m} u_{m}-\rho_{m} P_{m}\right)+\sqrt{\left(\rho_{+} P_{+}-\rho_{m} P_{m}\right)^{2}+4 \rho_{m} \rho_{+}\left(u_{+}-u_{m}\right)\left(u_{+}+P_{+}-u_{m} P_{m}\right)}}{2\left(\rho_{+}-\rho_{m}\right)}, \\
\beta_{1}(t)=\left(\left(\rho_{m}-\rho_{-}\right) \tau_{\delta_{1}}-\left(\rho_{m} u_{m}-\rho_{-} u_{-}\right)\right) t, \\
\beta_{2}(t)=\left(\left(\rho_{+}-\rho_{m}\right) \tau_{\delta_{2}}-\left(\rho_{+} u_{+}-\rho_{m}-u_{m}\right)\right) t,
\end{gathered}
$$

where $\tau_{\delta_{1}}$ and $\tau_{\delta_{2}}$ are respectively the propagating speed of $\delta S_{1}$ and $\delta S_{2}$, $\beta_{1}(t)$ and $\beta_{2}(t)$ are respectively the strength of $\delta S_{1}$ and $\delta S_{2}$.

It easy to see that $\tau_{\delta_{1}}>\tau_{\delta_{2}}$ which shows that $\delta S_{1}$ will overtake $\delta S_{2}$ at a point $\left(x_{1}, t_{1}\right)$ which is determined by

$$
\left\{\begin{array}{l}
x_{1}+\varepsilon=\tau_{\delta_{1}} t_{1}, \\
x_{1}-\varepsilon=\tau_{\delta_{1}} t_{1}
\end{array}\right.
$$

which yields

$$
\left(x_{1}, t_{1}\right)=\left(\frac{\left(u_{\delta_{1}}+u_{\delta_{2}}\right) \varepsilon}{u_{\delta_{1}}-u_{\delta_{2}}}, \frac{2 \varepsilon}{u_{\delta_{1}}-u_{\delta_{2}}}\right) .
$$

At the intersection point $\left(x_{1}, t_{1}\right)$, a new initial data is formed as follows

$$
\left.u\right|_{t_{1}}=\left\{\left.\begin{array}{ll}
u_{-}, & x<x_{1}, \\
u_{+}, & x>x_{1},
\end{array}\right|_{t_{1}}=\left\{\begin{array}{ll}
\rho_{-}, & x<x_{1}, \\
\rho_{+}, & x>x_{1},
\end{array}\right\}+\beta\left(t_{1}\right) \delta_{\left(x_{1}, t_{1}\right)},\right.
$$


where $\beta\left(t_{1}\right)=\beta_{1}\left(t_{1}\right)+\beta_{1}\left(t_{1}\right)$ is the sum of the strengths of the incoming delta shock wave $\delta S_{1}$ and $\delta S_{2}$. A new delta shock wave will generate after interaction and we denote it by $\delta S_{3}$, which is given by

$$
\left\{\begin{array}{l}
u(x, t)=u_{-}+\left(u_{+}-u_{-}\right) H, \\
\rho(x, t)=\rho_{-}+\left(\rho_{+}-\rho_{-}\right) H+\beta_{-}(t) D^{-}+\beta_{+}(t) D^{+},
\end{array}\right.
$$

where $H$ is the Heaviside function and $\beta(t)=\beta_{-}(t) D^{-}+\beta_{+}(t) D^{+}$is a split delta function. All of them are supported by the line $x=x_{1}+\left(t-t_{1}\right) \tau_{\delta_{3}}, \tau_{\delta_{3}}$ is the propagating speed of $\delta S_{3}$. Although they are supported by the same line, $D^{-}$is the delta measure on the set $\overline{R_{+}^{2}} \cap\left\{(x, t) \mid x \leq x_{1}+\left(t-t_{1}\right) \tau_{\delta_{3}}\right\}$ and $D^{+}$is the delta measure on the set $\overline{R_{+}^{2}} \cap\left\{(x, t) \mid x \geq x_{1}+\left(t-t_{1}\right) \tau_{\delta_{3}}\right\}$ respectively.

From (23), we obtain

$$
\begin{gathered}
\rho_{t}=\left(\rho_{+}-\rho_{-}\right)\left(-\tau_{\delta_{3}}\right) \delta+\beta_{-}^{\prime}(t) \delta+\beta_{+}^{\prime}(t) \delta-\tau_{\delta_{3}}\left(\beta_{-}(t)+\beta_{+}(t)\right) \delta^{\prime}, \\
(\rho u)_{x}=\left(\rho_{+} u_{+}-\rho_{-} u_{-}\right) \delta+\left(u_{-} \beta_{-}(t)+u_{+} \beta_{+}(t)\right) \delta^{\prime} .
\end{gathered}
$$

Substituting (24) and (25) into the first equation of (1), we obtain

$$
\beta(t)=\beta\left(t_{1}\right)+\left(\tau_{\delta_{3}}\left(\rho_{+}-\rho_{-}\right)-\left(\rho_{+} u_{+}-\rho_{-} u_{-}\right)\right)\left(t-t_{1}\right) .
$$

From (15), we get

$$
\tau_{\delta_{3}}=\frac{\left(2 \rho_{+} u_{+}+\rho_{+} P_{+}-2 \rho_{-} u_{-}-\rho_{-} P_{-}\right)+\sqrt{\left(\rho_{+} P_{+}-\rho_{-} P_{-}\right)^{2}+4 \rho_{-} \rho_{+}\left(u_{+}-u_{-}\right)\left(u_{+}+P_{+}-u_{-} P_{-}\right)}}{2\left(\rho_{+}-\rho_{-}\right)} .
$$

Case 2: $S+J$ and $\delta S$.

In this case, a shock wave followed by a contact discontinuity emits from $(-\varepsilon, 0)$ and a delta shock wave emits from $(\varepsilon, 0)$ (Figure 3). The propagating speed of the contact discontinuity is $\tau_{1}=u_{m}=u_{*}$, and the propagating speed of the delta shock wave satisfies the $\delta$-entropy condition $u_{+}-\frac{B \alpha}{\rho_{+}^{\alpha}}<u_{+}<\tau_{\delta_{1}}<u_{m}-\frac{B \alpha}{\rho_{m}^{\alpha}}<u_{m}$. It easy to see that $J$ will overtake $\delta S_{1}$ at $\left(x_{1}, t_{1}\right)$ which given by

$$
\left\{\begin{array}{l}
x_{1}+\varepsilon=u_{m} t_{1} \\
x_{1}-\varepsilon=\tau_{\delta_{1}} t_{1}
\end{array}\right.
$$

From (26), we get

$$
\left(x_{1}, t_{1}\right)=\left(\frac{\left(u_{\delta_{1}}+u_{m}\right) \varepsilon}{u_{m}-u_{\delta_{1}}}, \frac{2 \varepsilon}{u_{m}-u_{\delta_{1}}}\right) .
$$

The delta shock wave $\delta S_{1}$ will pass through $J$ with the same speed as before but the strength changes due to the difference between $\rho_{*}$ and $\rho_{m}$. We still denote it with $\delta S_{1}$ after the time $t_{1}$, and $\beta\left(t_{1}\right)=\left(\rho_{+}-\rho_{m}\right) \tau_{\delta_{1}}-\left(\rho_{+} u_{+}-\rho_{m} u_{m}\right)$.

From the $\delta$-entropy condition 




Figure 3. Interaction of $S+J$ and $\delta S$.

$$
u_{+}-\frac{B \alpha}{\rho_{+}^{\alpha}}<u_{+}<\tau_{\delta_{1}}<u_{*}-\frac{B \alpha}{\rho_{*}^{\alpha}}<u_{*},
$$

and the shock entropy condition

$$
u_{*}-\frac{B \alpha}{\rho_{*}^{\alpha}}<\tau_{1}<u_{-}-\frac{B \alpha}{\rho_{-}^{\alpha}},
$$

we know that $S$ will overtake $\delta S_{1}$ at $\left(x_{2}, t_{2}\right)$ which satisfies

$$
\left\{\begin{array}{l}
x_{2}+\varepsilon=\tau_{1} t_{2} \\
\left(x_{2}-x_{1}\right)=\tau_{\delta_{1}}\left(t_{2}-t_{1}\right)
\end{array}\right.
$$

thus

$$
\left(x_{2}, t_{2}\right)=\left(2 \varepsilon, \frac{3 \varepsilon}{\tau_{1}}\right) .
$$

The new initial data will be formulated at $\left(x_{2}, t_{2}\right)$ as follows

$$
\left.u\right|_{t_{2}}=\left\{\left.\begin{array}{ll}
u_{-}, & x<x_{2}, \\
u_{+}, & x>x_{2},
\end{array} \quad \rho\right|_{t_{1}}=\left\{\begin{array}{ll}
\rho_{-}, & x<x_{2}, \\
\rho_{+}, & x>x_{2},
\end{array}\right\}+\beta\left(t_{2}\right) \delta_{\left(x_{2}, t_{2}\right)},\right.
$$

$\beta\left(t_{2}\right)=\left(\rho_{+}-\rho_{*}\right) \tau_{\delta_{1}}-\left(\rho_{+} u_{+}-\rho_{*} u_{*}\right)$ denotes the strength of $\delta S_{1}$ at the time $t_{2}$ and from $u_{*}=u_{m}$ and $u_{*}=u_{-}+\frac{B}{\rho_{*}^{\alpha}}-\frac{B}{\rho_{-}^{\alpha}}$ we can determine the value of $\left(u_{*}, \rho_{*}\right)$.

A new delta shock wave will be generated after the interaction of $S$ and $\delta S_{1}$, denoted by $\delta S_{2}$ here. It satisfies that

$$
\left\{\begin{array}{l}
u(x, t)=u_{-}+\left(u_{+}-u_{-}\right) H, \\
\rho(x, t)=\rho_{-}+\left(\rho_{+}-\rho_{-}\right) H+\beta_{-}(t) D^{-}+\beta_{+}(t) D^{+} .
\end{array}\right.
$$

The Heaviside function $H$ and the split delta function $\beta(t) D=\beta_{-}(t) D^{-}+\beta_{+}(t) D^{+}$are supported by the line $x=x_{2}+\left(t-t_{2}\right) \tau_{\delta_{2}}$, $\tau_{\delta_{2}}$ is the propagating speed of $\delta S_{2}$. From (31), similarly with the above case we obtain that the strength of $\delta S_{2}$ after the interaction of $S$ and $\delta S_{1}$ is

$$
\begin{gathered}
\beta(t)=\beta\left(t_{2}\right)+\left(\tau_{\delta_{2}}\left(\rho_{+}-\rho_{-}\right)-\left(\rho_{+} u_{+}-\rho_{-} u_{-}\right)\right)\left(t-t_{2}\right), \\
\beta_{-}(t)=\frac{\tau_{\delta_{2}}-u_{+}}{u_{-}-u_{+}} \beta(t),
\end{gathered}
$$




$$
\beta_{+}(t)=\frac{u_{-}-\tau_{\delta_{2}}}{u_{-}-u_{+}} \beta(t) .
$$

As $t>t_{2}$, the delta shock wave $\delta S_{2}$ will propagate with the invariant speed $\tau_{\delta_{2}}$ which is given by (15) with $\left(u_{+}, \rho_{+}\right)$and $\left(u_{-}, \rho_{-}\right)$as its right and left state. Furthermore, from the condition $u_{-}<u_{-}-\frac{B}{\rho_{-}^{\alpha}}<u_{m}<u_{-}$we know the $\delta$ -entropy condition for the new delta shock wave $\tau_{\delta_{2}}$ holds which shows $\tau_{\delta_{2}}$ is an overcompresive wave.

Case 3: $R+J$ and $\delta S$.

When $t$ is small enough, the solution of the initial value problem (1) (3) and (5) can be described by (Figure 4 )

$$
\left(u_{-}, \rho_{-}\right)+R+\left(u_{*}, \rho_{*}\right)+J+\left(u_{m}, \rho_{m}\right)+\delta S_{1}+\left(u_{+}, \rho_{+}\right) .
$$

Similar with the above case, the contact discontinuity $J$ will overtake the delta shock wave $\delta S_{1}$ at the point $\left(x_{1}, t_{1}\right)$ given by (27). The delta shock wave $\delta S_{1}$ will pass through $J$ with the same speed as before but the strength changes due to the difference between $\rho_{*}$ and $\rho_{m}$. We still denote it with $\delta S_{1}$ after the time $t_{1}$, and $\beta\left(t_{1}\right)=\left(\rho_{+}-\rho_{m}\right) \tau_{\delta_{1}}-\left(\rho_{+} u_{+}-\rho_{m} u_{m}\right)$. Since the propagation speed of wave front in the rarefaction wave is $\lambda_{1}=u_{*}-\frac{B \alpha}{\rho_{*}^{\alpha}}$ and that of the delta shock wave $\delta S_{1}$ satisfies the $\delta$-entropy condition

$$
u_{+}-\frac{B \alpha}{\rho_{+}^{\alpha}}<u_{+}<\tau_{\delta_{1}}<u_{*}-\frac{B \alpha}{\rho_{*}^{\alpha}}<u_{*},
$$

it is shown that $R$ will interact with $\delta S_{1}$ at $\left(x_{2}, t_{2}\right)$ which is determined by

$$
\left\{\begin{array}{l}
x_{2}+\varepsilon=\lambda_{1} t_{2}, \\
\left(x_{2}-x_{1}\right)=\tau_{\delta_{1}}\left(t_{2}-t_{1}\right),
\end{array}\right.
$$

it follows that $\left(x_{2}, t_{2}\right)=\left(\frac{\left(\lambda_{1}+\tau_{\delta_{1}}\right) \varepsilon}{\lambda_{1}-\tau_{\delta_{1}}}, \frac{2 \varepsilon}{\lambda_{1}-\tau_{\delta_{1}}}\right)$. The strength of $\delta S_{1}$ at $\left(x_{2}, t_{2}\right)$ can be calculated by $\beta\left(t_{2}\right)=\left(\left(\rho_{+}-\rho_{*}\right) \tau_{\delta_{1}}-\left(\rho_{+} u_{+}-\rho_{*} u_{*}\right)\right) t_{2}$. At the same time, a new delta shock wave $\delta S_{2}$ with varying speed is generated. Here we use $\Gamma: x=x(t), t \geq t_{2}$ to express the curve of $\delta S_{2}$ and it is given in the following form

$$
\begin{aligned}
u(x, t)= \begin{cases}\frac{1}{1-\alpha} \frac{x-\varepsilon}{t}+\frac{\alpha}{1-\alpha}\left(-u_{*}+\frac{B}{\rho_{*}^{\alpha}}\right), & x<x(t), \\
u_{+}, & x>x(t),\end{cases} \\
\rho(x, t)=\left\{\begin{array}{ll}
(B(1-\alpha))^{\frac{1}{\alpha}}\left(\frac{x-\varepsilon}{t}-u_{*}+\frac{B}{\rho_{*}^{\alpha}}\right)^{-\frac{1}{\alpha}}, & x<x(t), \\
\rho_{+} & x>x(t),
\end{array}\right\} \\
+\beta_{-}(t) D_{\Gamma}^{-}+\beta_{+}(t) D_{\Gamma}^{+},
\end{aligned}
$$




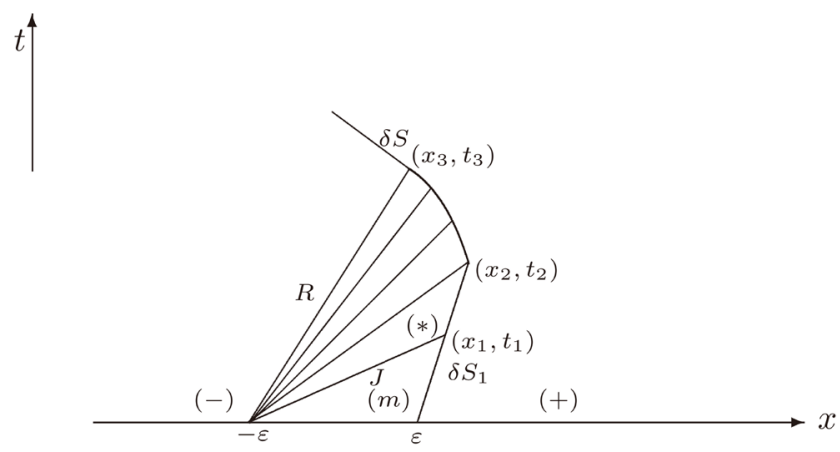

Figure 4. Interaction of $R+J$ and $\delta S_{1}$.

where $\beta(t) D_{\Gamma}=\beta_{-}(t) D_{\Gamma}^{-}+\beta_{+}(t) D_{\Gamma}^{+}$is a split delta function on the new delta shock, and $\beta(t)=\beta_{-}(t)+\beta_{+}(t)$ is the strength of the new delta shock at the time $t$.

When $u_{+}>u_{-}$, the delta shock wave cannot penetrate the rarefaction wave; when $u_{+} \leq u_{-}$, the delta shock wave can penetrate the rarefaction wave completely.

\section{Conclusions}

Now we construct the unique solution of the elementary wave interactions and get the following main conclusion. Using the characteristic analysis method, i.e., by analyzing the elementary wave curves in the phrase plane, we get the unique solution of the initial problem (1) with the state equation (3) and the initial values (4). We observe that the elementary wave interactions have a much simpler structure for Temple class than general systems of conservation laws since the wave interaction of the same family does not generate wave of other families for Temple systems. It is important to study the elementary waves interactions for (1) not only because of their significance in practical applications in the traffic flow system for the generalized Chaplygin gas, but also because of their basic role as building blocks for the general mathematical theory of the traffic flow system.

Theorem 4.1 The Riemann solutions of the initial value problem (1) (3) with the initial data (4) are constructed which are stable under such small perturbation on the initial data.

\section{Funding}

Supported by the Foundation for Young Scholars of Shandong University of Technology (No. 115024).

\section{Conflicts of Interest}

The author declares no conflicts of interest regarding the publication of this paper.

\section{References}

[1] Daganzo, C. (1995) Requiem for Second Order Fluid Approximations of Traffic Flow. Transportation Research Part B: Methodological, 29, 277-286. 
https://doi.org/10.1016/0191-2615(95)00007-Z

[2] Zhang, H.M. (2002) A Non-Equilibrium Traffic Model Devoid of Gas-Like Behavior. Transportation Research Part B: Methodological, 36, 275-290.

https://doi.org/10.1016/S0191-2615(00)00050-3

[3] Herty, M. and Rascle, M. (2006) Coupling Conditions for a Class of Second-Order Models for Traffic Flow. SIAM Journal on Applied Mathematics, 38, 595-616. https://doi.org/10.1137/05062617X

[4] Klar, A., Greenberg, J.M. and Rascle, M. (2003) Congestion o n Multilane Highways. SIAM Journal on Applied Mathematics, 63, 818-833. https://doi.org/10.1137/S0036139901396309

[5] Greenberg, J.M. (2001) Extensions and Amplifications of a Traffic Model of Aw and Rascle. SIAM Journal on Applied Mathematics, 62, 729-745. https://doi.org/10.1137/S0036139900378657

[6] Garavello, M. and Piccoli, B. (2006) Traffic Flow on a Road Network Using the Aw-Rascle Model. Comm. Partial Differential Equations, 31, 243-275. https://doi.org/10.1080/03605300500358053

[7] Moutari, S. and Rascle, M. (2007) A Hybrid Lagrangian Model Based on the Aw-Rascle Traffic Flow Model. SIAM Journal on Applied Mathematics, 68, 413-436. https://doi.org/10.1137/060678415

[8] Berthelin, F., Degond, P., Delitata, M. and Rascle, M. (2008) A Model for the Formation and Evolution of Traffic Jams. Archive for Rational Mechanics and Analysis, 187, 185-220. https://doi.org/10.1007/s00205-007-0061-9

[9] Shen, C. and Sun, M.N. (2010) Formation of Delta-Shocks and Vacuum States in the Vanishing Pressure Limit of Solutions to the Aw-Rascle Model. Journal of Differential Equations, 249, 3024-3051. https://doi.org/10.1016/j.jde.2010.09.004

[10] Sun, M.N. (2009) Interactions of Elementary Waves for the Aw-Rascle Model. SIAM Journal on Applied Mathematics, 69, 1542-1558. https://doi.org/10.1137/080731402

[11] Sun, M.N. (2011) A Note on the Interactions of Elementary Waves for the AR Traffic Flow Model without Vacuum. Acta Mathematica Scientia, 31B, 1503-1512. https://doi.org/10.1016/S0252-9602(11)60336-6

[12] Wang, G.D. (2014) The Riemann Problem for Aw-Rascle Traffic Flow with Negative Pressure. Chinese Annals of Mathematics, Series A, 35, 73-82.

[13] Liu, Y.J. and Sun, W.H. (2018) Wave Interactions and Stability of Riemann Solutions of the Aw? CRascle Model for Generalized Chaplygin Gas. Acta Applicandae Mathematicae, 154, 95-109. https://doi.org/10.1007/s10440-017-0135-0

[14] Temple, B. (1983) Systems of Conservation Laws with Coinciding Shock and Rarefaction Curves. Contemporary Mathematics, 17, 143-151. https://doi.org/10.1090/conm/017/16 